\title{
Multiple Dirac particles in AA-stacked graphite and multilayers of graphene
}

\author{
I. Lobato and B. Partoens* \\ University of Antwerp, Department of Physics, Groenenborgerlaan 171, B-2020 Antwerp, Belgium
}

(Received 19 April 2010; revised manuscript received 15 February 2011; published 21 April 2011)

\begin{abstract}
Using the tight-binding formalism we show that in the recently experimentally realized AA-stacked graphite in essence two types of massless relativistic Dirac particles are present with a different effective speed of light. We also investigate how the electronic structure evolves from a single graphene sheet into AA-stacked graphite. It is shown that in contrast to AB-stacked graphene layers, the spectrum of AA-stacked graphene layers can be considered as a superposition of single-layer spectra and only particles with a linear spectrum at the Fermi energy around the $K$ point are present. From the evolution of the band overlap we show that 6 multilayers of AA-stacked graphene already behave as AA-stacked graphite. The evolution of the effective speeds of light of the Dirac particles to their bulk values shows exactly the same behavior. The tight-binding parameters we use to describe AA-stacked graphite and multilayers of graphene are obtained by ab initio calculations.
\end{abstract}

DOI: 10.1103/PhysRevB.83.165429

PACS number(s): 73.22.Pr, 68.65.Pq, 81.05.uf

\section{INTRODUCTION}

Graphite consists of van der Waals coupled graphene layers. Graphene is a layer of carbon atoms with hexagonal symmetry. In graphite every other layer of graphene is shifted in the horizontal plane, leading to the well-known $\mathrm{AB}$ or Bernal stacking. ${ }^{1}$ In nature also the $\mathrm{ABC}$ stacked graphite can be found, ${ }^{2}$ while AA-stacked graphite was, until recently, only found in intercalated graphite, for example with $\mathrm{Li}^{3}$ But in 2008, the growth of AA-stacked graphite on the (111) surface of diamond was reported. ${ }^{4}$ Very recently, it was also shown that bilayer graphene often exhibits AA-stacking and that it is very hard to distinguish it from a single graphene layer. ${ }^{5}$

Recently, there has been an increased amount of experimental and theoretical interest in the electronic properties of ultrathin graphite films. It is well established now that the charge carriers in a single layer of graphene around the Fermi energy are massless Dirac fermions with a linear energy spectrum. ${ }^{6,7}$ Remarkably, Dirac fermions (holes) are also present in AB-stacked graphite at the $H$ point of the Brillouin zone and coexist with normal (massive) electrons located at the $K$ point as was recently proved by angleresolved photoemission spectroscopy experiments on ABstacked graphite. ${ }^{8}$

Now that AA-stacked graphite and a few layers of graphene can be realized experimentally, it is interesting to investigate whether Dirac fermions are also present in AA-stacked graphene multilayers and graphite. The electronic structure of AA-stacked graphite has been studied before using firstprinciples density functional theory calculations ${ }^{9}$ and within the tight-binding approach. ${ }^{10,11}$ These works discuss the full band structure and compare it with the one for Bernal and $\mathrm{ABC}$ stacked graphite. Although few, there are works that study the electronic structure of AA-stacked graphene bilayers and multilayers. ${ }^{12,13}$ In contrast to these previous studies, in this work we focus on the nature of the charge carriers around the Fermi energy and study the evolution of the electronic structure as a function of the number of AA-stacked graphene layers. To realize this, we apply the tight-binding approach. The tightbinding parameters are obtained by means of first-principles density functional theory calculations. The evolution of the electronic structure as a function of the number of AB-stacked graphene layers was studied before and it was shown that starting from around $10 \mathrm{AB}$-stacked graphene multilayers the band overlap corresponds with the one of Bernal graphite. ${ }^{14}$

This paper is further organized as follows. In the next section the results of the tight-binding model for AA-stacked graphite are summarized with a focus on the dispersion relation for the charge carriers around the Fermi energy. In Sec. III a tight-binding model for AA-stacked graphene multilayers is presented and the evolution of the electronic structure from from AA-stacked graphene multilayers to AA-stacked graphite is discussed. Our conclusions are summarized in Sec. IV. Our tight-binding parameters are obtained from first-principles density functional theory calculations, and the approach is presented in the Appendix.

\section{AA-STACKED GRAPHITE}

Figure 1(a) shows schematically the crystal structure of AA-stacked graphite and AA-stacked graphene multilayers. The shaded area indicates the primitive cell. The translational vectors of the crystal structure are $\vec{a}_{1}=a(\sqrt{3} / 2,1 / 2,0), \vec{a}_{2}=$ $a(\sqrt{3} / 2,-1 / 2,0)$, and $\vec{a}_{3}=c_{0}(0,0,1)$. The in-plane lattice parameter is $a=\sqrt{3} a_{0}$, with $a_{0}=1.41 \AA$ the nearest-neighbor distance. The distance between two layers is $c_{0}=3.63 \AA$. These values have been calculated by first-principles density functional theory calculations (see Appendix). The corresponding Brillouin zone with the name labels for the highsymmetry points for AA-stacked graphite is shown in Fig. 1(b). The reciprocal lattice is given by $\vec{b}_{1}=\frac{2 \pi}{a}(1 / \sqrt{3}, 1,0), \vec{b}_{2}=$ $\frac{2 \pi}{a}(1 / \sqrt{3},-1,0)$, and $\vec{b}_{3}=\frac{2 \pi}{c_{0}}(0,0,1)$. For the case of AAstacked multilayers there is no periodicity in the $z$ direction, and the corresponding Brillouin zone is shown in Fig. 1(c).

The electronic band structure as obtained within a tightbinding approach is given by ${ }^{10}$

$$
E=\gamma_{1} \Gamma\left(k_{z}\right)+\gamma_{5}\left[\Gamma\left(k_{z}\right)^{2}-2\right] \pm\left[\gamma_{0}+\gamma_{4} \Gamma\left(k_{z}\right)\right]\left|f\left(k_{x}, k_{y}\right)\right|,
$$

where $f\left(k_{x}, k_{y}\right)=e^{i k_{x} a / \sqrt{3}}+2 e^{-i k_{x} a /(2 \sqrt{3})} \cos \left(k_{y} a / 2\right) \quad$ and $\Gamma\left(k_{z}\right)=2 \cos \left(k_{z} c_{0}\right)$. The definition of the tight-binding parameters is chosen as in Refs. 14 and 15. As shown in Refs. 9-11, the Fermi energy crosses the energy bands close 
(a)

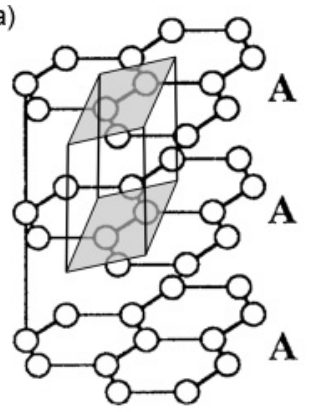

(b)

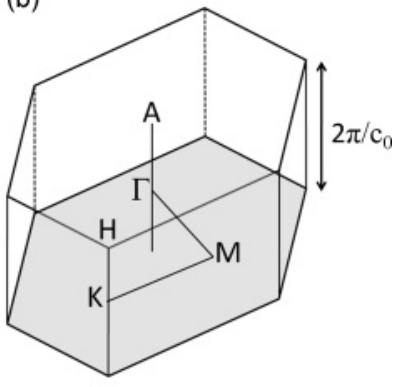

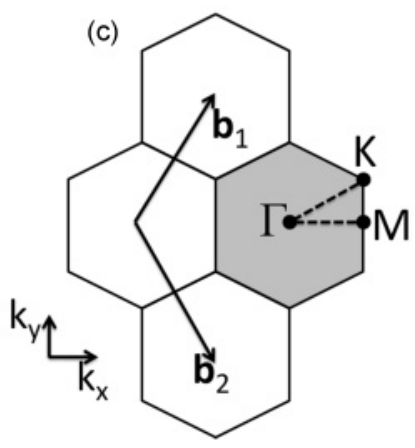

FIG. 1. (a) The crystal structure of AA-stacked graphite, (b) the corresponding Brillouin zone, and (c) the Brillouin zone for a finite number of stacked graphene layers.

to the $H K H$ line of the Brillouin zone, just as in AB-stacked graphite. The band structure along this line and in the $\Gamma$ and $A$ directions is shown in Fig. 2(a). The values of the tight-binding parameters are obtained by fitting the tight-binding model to $a b$ initio calculations as explained in the Appendix. Their values are listed in Table I.

The Fermi energy is close to zero (but not exactly; see Ref. 10), resulting in a Fermi surface composed of a main hole pocket around the $K$ point and two smaller pockets of electrons centered at the two $H$ points as shown in Fig. 2(b). The energy at the $H$ point is $E_{H}=-2 \gamma_{1}+2 \gamma_{5}$ and at the $K$ point $E_{K}=$ $2 \gamma_{1}+2 \gamma_{5}$, resulting in a band overlap of $4 \gamma_{1}=0.8638 \mathrm{eV}$.

The dispersion of the energy bands around this $H K H$ line, which determines the nature of the charge carriers around the Fermi energy, can be obtained by an expansion of the energy expression Eq. (1) in $\vec{k}$. We find to first order

$$
\left|f\left(k_{x}, k_{y}\right)\right|=\alpha d k
$$

where $\alpha=\frac{\sqrt{3}}{2} a$ and $d k$ is the distance from the $H K H$ line in the direction of the $A \Gamma A$ line. As a consequence, the energy dispersion around the $H K H$ line,

$$
E=\gamma_{1} \Gamma+\gamma_{5}\left[\Gamma\left(k_{z}\right)^{2}-2\right] \pm\left[\gamma_{0}+\gamma_{4} \Gamma\left(k_{z}\right)\right] \alpha d k,
$$

shows a linear dispersion in the direction of the $A \Gamma A$ line for all $k_{z}$ values. There are no normal charge carriers with a parabolic spectrum as in AB-stacked graphite in the $K$ point. The corresponding effective speed of light of these massless Dirac particles is

$$
c=\frac{\alpha}{\hbar}\left[\gamma_{0}+\gamma_{4} \Gamma\left(k_{z}\right)\right]
$$
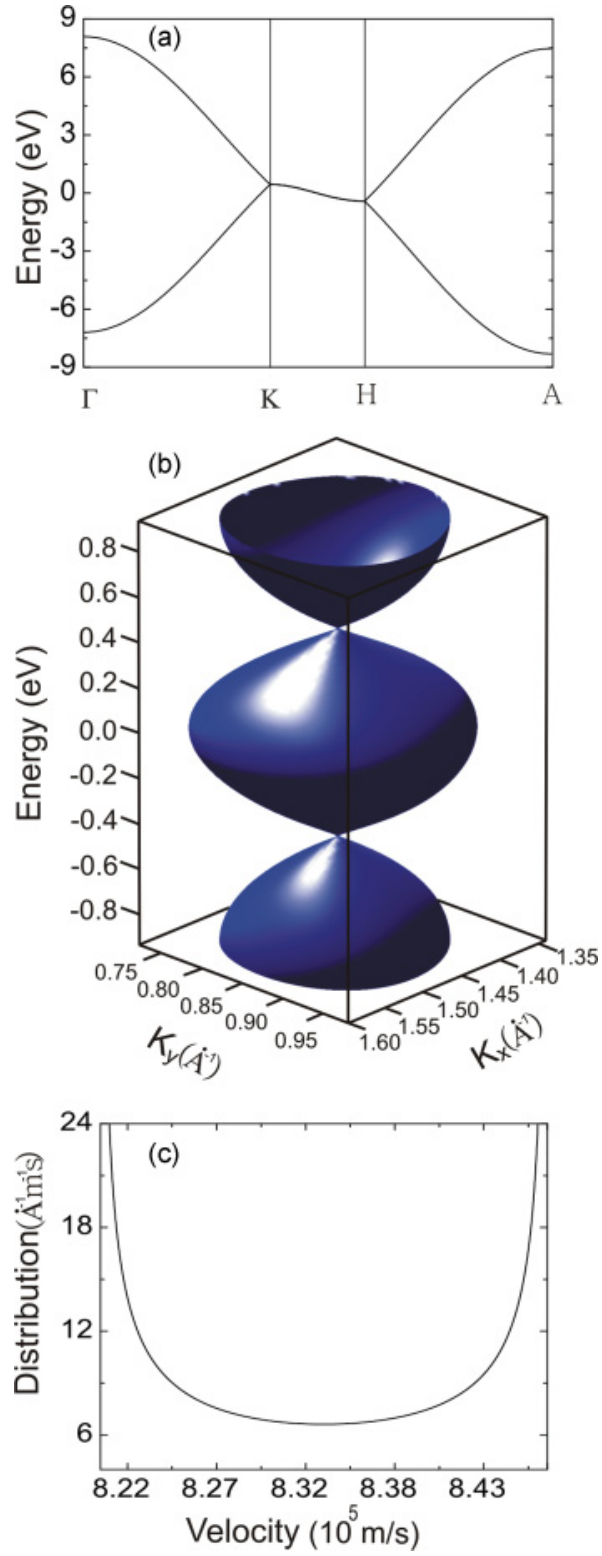

FIG. 2. (Color online) (a) Band structure of AA-stacked graphite along ГКНА. (b) Fermi surface of AA-stacked graphite, composed of a pocket of holes centered around the $K$ point, and two half pockets of electrons around the $H$ points. (c) The distribution of the effective speeds of light along the $H K H$ line in AA-stacked graphite.

The velocities are not evenly distributed, but the distribution is given by

$$
\rho(c)=\frac{1}{\pi c_{0}}\left[\left(\frac{2 \gamma_{4}}{\hbar}\right)^{2}-\left(c-\frac{\alpha \gamma_{0}}{\hbar}\right)^{2}\right]^{-1 / 2}
$$

and is shown in Fig. 2(c). The two peaks arise from states around the $K$ and $H$ points. As the charge carrier pockets are located around these $K$ and $H$ points, we find that there exist in essence two types of massless Dirac particles in AAstacked graphite, with a (minimal) effective speed of light $c=$ $\frac{\alpha}{\hbar}\left(\gamma_{0}+2 \gamma_{4}\right)=8.20 \times 10^{5} \mathrm{~m} / \mathrm{s}$ at the $K$ point, and a (maximal) effective speed of light $c=\frac{\alpha}{\hbar}\left(\gamma_{0}-2 \gamma_{4}\right)=8.47 \times 10^{5} \mathrm{~m} / \mathrm{s}$ at the $H$ point. For comparison, the effective speed of light of 
the Dirac particles in graphene in the $K$ point is $c=\frac{\alpha}{\hbar} \gamma_{0}=$ $8.34 \times 10^{5} \mathrm{~m} / \mathrm{s}$.

\section{AA-STACKED GRAPHENE MULTILAYERS}

In order to discuss the evolution of the electronic structure of AA-stacked graphene multilayers, we first construct the tightbinding Hamiltonian. The system of AA-stacked graphene multilayers is periodic in the $x$ and $y$ direction, and contains $2 N$ atoms in the unit cell, with $N$ the number of graphene layers. Therefore we define two tight-binding Bloch functions per layer graphene:

$$
\begin{aligned}
& \Phi_{\vec{k}}^{A_{i}}(\vec{r})=\frac{1}{\sqrt{N_{c}}} \sum_{\vec{A}_{i}} e^{i \vec{k} \cdot \vec{r}_{A_{i}}} \varphi_{A}\left(\vec{r}-\vec{r}_{A_{i}}\right), \\
& \Phi_{\vec{k}}^{B_{i}}(\vec{r})=\frac{1}{\sqrt{N_{c}}} \sum_{\vec{B}_{i}} e^{i \vec{k} \cdot \vec{r}_{B_{i}}} \varphi_{B}\left(\vec{r}-\vec{r}_{B_{i}}\right),
\end{aligned}
$$

where the index $i$ runs over the number of layers and $N_{c}$ is the number of considered unit cells. The resulting tight-binding Hamiltonian for $N$ layers is the $2 N \times 2 N$ matrix

$$
\begin{aligned}
H & =\left[\begin{array}{ccccc}
\left\langle\Phi_{\vec{k}}^{A_{1}}|H| \Phi_{\vec{k}}^{A_{1}}\right\rangle & \left\langle\Phi_{\vec{k}}^{A_{1}}|H| \Phi_{\vec{k}}^{B_{1}}\right\rangle & \left\langle\Phi_{\vec{k}}^{A_{1}}|H| \Phi_{\vec{k}}^{A_{2}}\right\rangle & \cdots & \left\langle\Phi_{\vec{k}}^{A_{1}}|H| \Phi_{\vec{k}}^{B_{N}}\right\rangle \\
\left\langle\Phi_{\vec{k}}^{B_{1}}|H| \Phi_{\vec{k}}^{A_{1}}\right\rangle & \left\langle\Phi_{\vec{k}}^{B_{1}}|H| \Phi_{\vec{k}}^{B_{1}}\right\rangle & \left\langle\Phi_{\vec{k}}^{B_{1}}|H| \Phi_{\vec{k}}^{A_{2}}\right\rangle & \cdots & \left\langle\Phi_{\vec{k}}^{B_{1}}|H| \Phi_{\vec{k}}^{B_{N}}\right\rangle \\
\vdots & \vdots & \vdots & \ddots & \vdots \\
\left\langle\Phi_{\vec{k}}^{B_{N_{l}}}|H| \Phi_{\vec{k}}^{A_{1}}\right\rangle & \left\langle\Phi_{\vec{k}}^{B_{N_{l}}}|H| \Phi_{\vec{k}}^{B_{1}}\right\rangle & \left\langle\Phi_{\vec{k}}^{B_{N_{l}}}|H| \Phi_{\vec{k}}^{A_{2}}\right\rangle & \cdots & \left\langle\Phi_{\vec{k}}^{B_{N}}|H| \Phi_{\vec{k}}^{B_{N}}\right\rangle
\end{array}\right] \\
= & {\left[\begin{array}{cc|cc|cc|cc|c}
0 & \gamma_{0} f & \gamma_{1} & \gamma_{4} f & \gamma_{5} & 0 & 0 & 0 & \cdots \\
\hline \gamma_{o} f^{*} & 0 & \gamma_{4} f^{*} & \gamma_{1} & 0 & \gamma_{5} & 0 & 0 & \cdots \\
\hline \gamma_{1} & \gamma_{4} f & 0 & \gamma_{0} f & \gamma_{1} & \gamma_{4} f & \gamma_{5} & 0 & \cdots \\
\gamma_{4} f^{*} & \gamma_{1} & \gamma_{0} f^{*} & 0 & \gamma_{4} f^{*} & \gamma_{1} & 0 & \gamma_{5} & \cdots \\
\hline \vdots & \vdots & \vdots & \vdots & \vdots & \vdots & \vdots & \vdots
\end{array}\right] . }
\end{aligned}
$$

Again the values of the tight-binding parameters are obtained by comparing and fitting the electronic structure to $a b$ initio results of which the details are given in the Appendix. The values are also listed in Table I, which clearly shows that they are almost constant as a function of the number of layers.

The fundamental electronic character of the charge carriers around the Fermi energy in a single and two AB-stacked graphene layers is fundamentally different. While a single graphene layer has a linear spectrum at the Fermi energy around the $K$ point with a corresponding effective speed of light of $c=\frac{\alpha}{\hbar} \gamma_{0}=8.34 \times 10^{5} \mathrm{~m} / \mathrm{s}$, the system of two AB-stacked graphene layers has a parabolic spectrum at the Fermi energy around the $K$ point that goes over into a linear dispersion away

TABLE I. Tight-binding parameters for AA-stacked graphite and $N_{l}$ numbers of AA-stacked graphene layers obtained by firstprinciples calculations using values of the energy and its derivative at the $K$ point. Values of the four tight-binding parameters for $N_{l}=1$, $N_{l}=2, N_{l}=3$, and graphite, expressed in $\mathrm{eV}$.

\begin{tabular}{lcccc}
\hline \hline TB & Value for & Value for & Value for & Value for \\
Parameter & $N_{l}=1$ & $N_{l}=2$ & $N_{l}=3$ & AA Graphite \\
\hline$\gamma_{0}$ & 2.5891 & 2.5890 & 2.5889 & 2.5875 \\
$\gamma_{1}$ & 0 & 0.2174 & 0.2171 & 0.2159 \\
$\gamma_{4}$ & 0 & -0.0206 & -0.0206 & -0.0206 \\
$\gamma_{5}$ & 0 & 0 & 0.0024 & 0.0058 \\
\hline \hline
\end{tabular}

from the $K$ point. Therefore let us first look at how the spectrum changes from graphene to two AA-stacked graphene layers. Figure 3(a) shows the energy spectrum for two AA-stacked graphene layers and Fig. 3(b) is a zoom close to the $K$ point in the direction of the $\Gamma$ and $M$ points. In contrast to two AB-stacked graphene layers, the spectrum can now be considered as the combination of two shifted linear spectra. Figure 3(c) shows the highest valence band for this system around the $K$ point. While for a single graphene layer there is only a linear spectrum in one point in the Brillouin zone, there is now a whole ring in the Brillouin zone where Dirac particles with a linear spectrum are present. The inner and outer radial velocities of the states on this ring are shown in Fig. 3(d). Note that these velocities oscillate around the values $8.20 \times 10^{5} \mathrm{~m} / \mathrm{s}$ and $8.47 \times 10^{5} \mathrm{~m} / \mathrm{s}$, which are very close to the velocities of the same bands in the $K$ point, marked by the two asterisks in Fig. 3(b). Therefore we will use later the velocities in the $K$ point as good approximations for the effective speed of light values around the Fermi energy. The oscillating velocities in Fig. 3(d) reach their maximal and minimal values three times and thus show clearly the trigonal warping effect away from the $K$ point.

Further increasing the number of AA-stacked graphene layers changes the electronic spectrum in a similar way. Figure 4 shows an illustrative example for 5 AA-stacked graphene layers. Figure 4(a) shows the band structure along the $\Gamma K M \Gamma$ line, and Fig. 4(b) is a zoom around the $K$ point. Now five crossing points can be observed around the Fermi energy. 

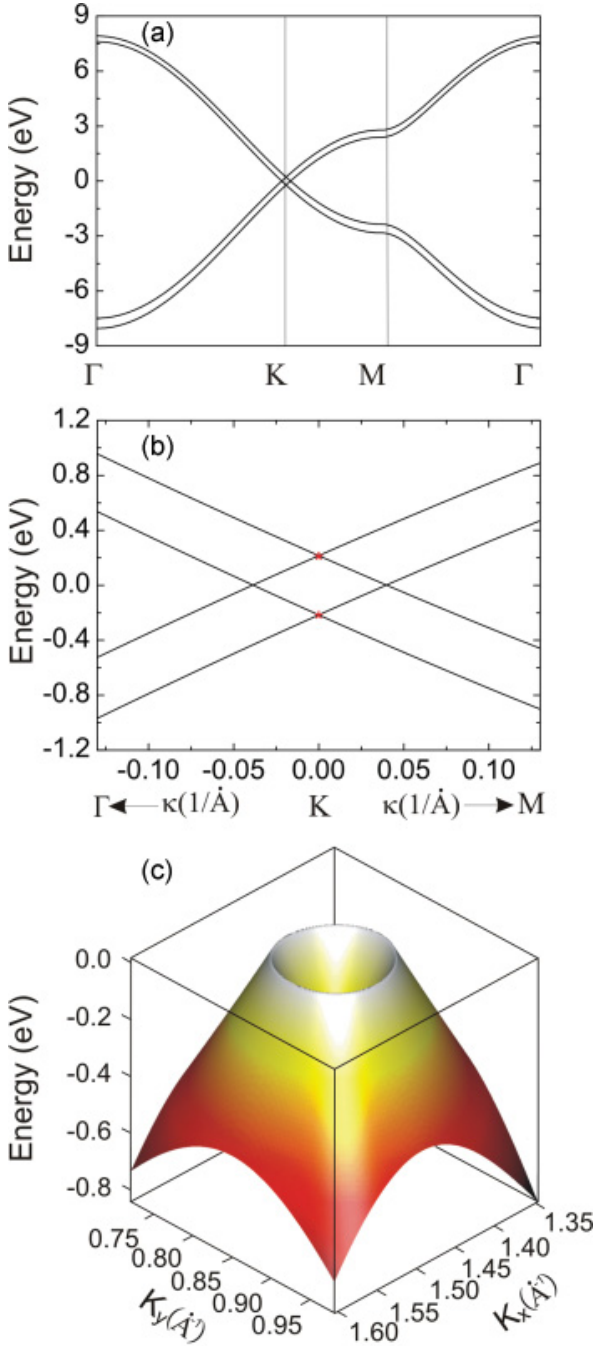

(d)

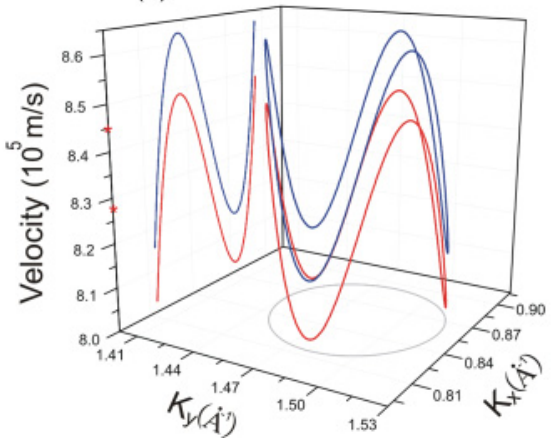

FIG. 3. (Color online) (a) Band structure of two AA-stacked graphene layers along $Г K M Г$. (b) Zoom of this band structure around the $K$ point. (c) Highest valence band of this system around the $K$ point. (d) The inner (blue curve) and outer (red curve) radial velocity of the states on the ring in (c).

Figure 4(c) shows again the highest valence band for this system around the $K$ point. Now two rings can be observed, as well as a cone at the $K$ point where Dirac particles are present. With increasing number of layers, the number of such rings increases, and finally, in the limit of AA-stacked graphite, they will evolve into the Fermi surface spheres of Fig. 2(b).
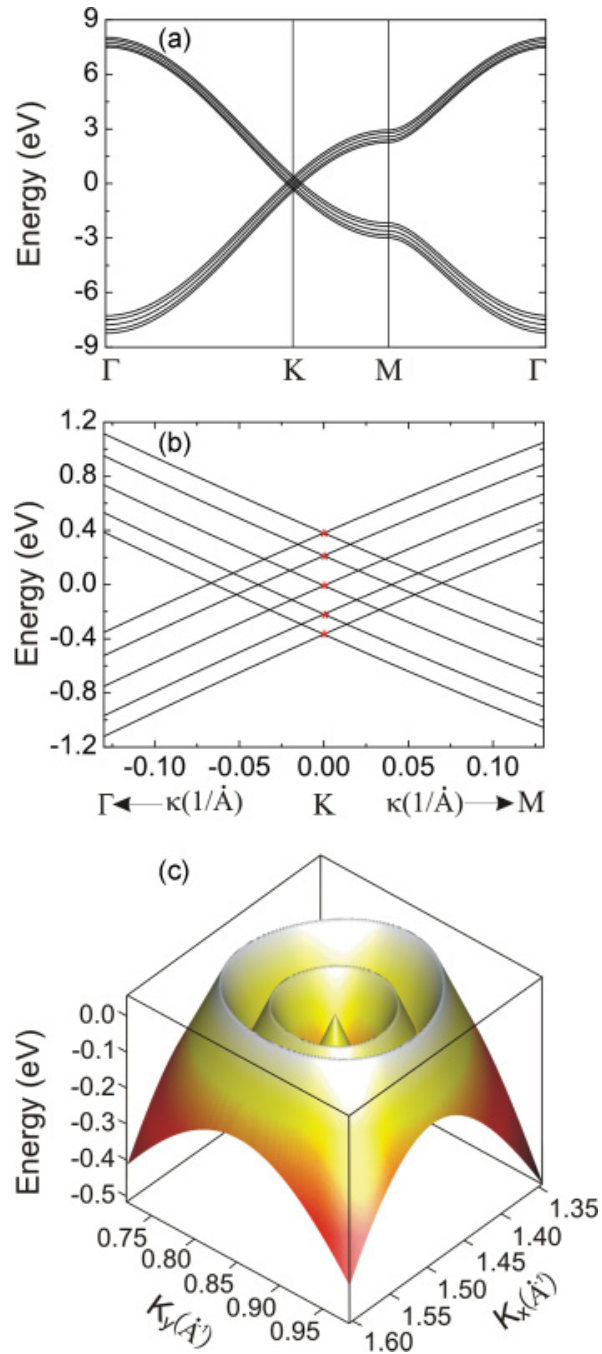

FIG. 4. (Color online) (a) Band structure of five AA-stacked graphene layers along $Г К М Г$. (b) Zoom of this band structure around the $K$ point. (c) Highest valence band of this system around the $K$ point.

Let us now investigate in more detail the evolution of the electronic structure from a single graphene layer, over multiple AA-stacked graphene layers, to the limit of AAstacked graphite. In Ref. 14 it was shown that from around 10 layers of $\mathrm{AB}$-stacked graphene layers, the difference in band overlap with AB-stacked graphite is smaller than $10 \%$. In order to see how the energy levels at the $K$ point for a finite number of AA-stacked layers evolve into the band structure of AA-stacked graphite along the $H K H$ edge [see Fig. 2(a)], we show in Fig. 5 a plot of the energy levels at the $K$ point, in increasing order and equally distributed over the interval $\left[0, \pi / c_{0}\right]$ for 6,10 , and 20 layers, together with the band structure of AA-stacked graphite along the $K H$ line. It is clear that the energies at the $K$ point of the multilayer systems evolve into the band structure of AA-stacked graphite. This allows us to approximate the band overlap in the finite-layer system by taking the difference between the maximum and minimum value of the energy at the $K$ point. This evolution of the band overlap as function of the number of layers is shown in Fig. 6. We observe that starting from 6 layers, the band 


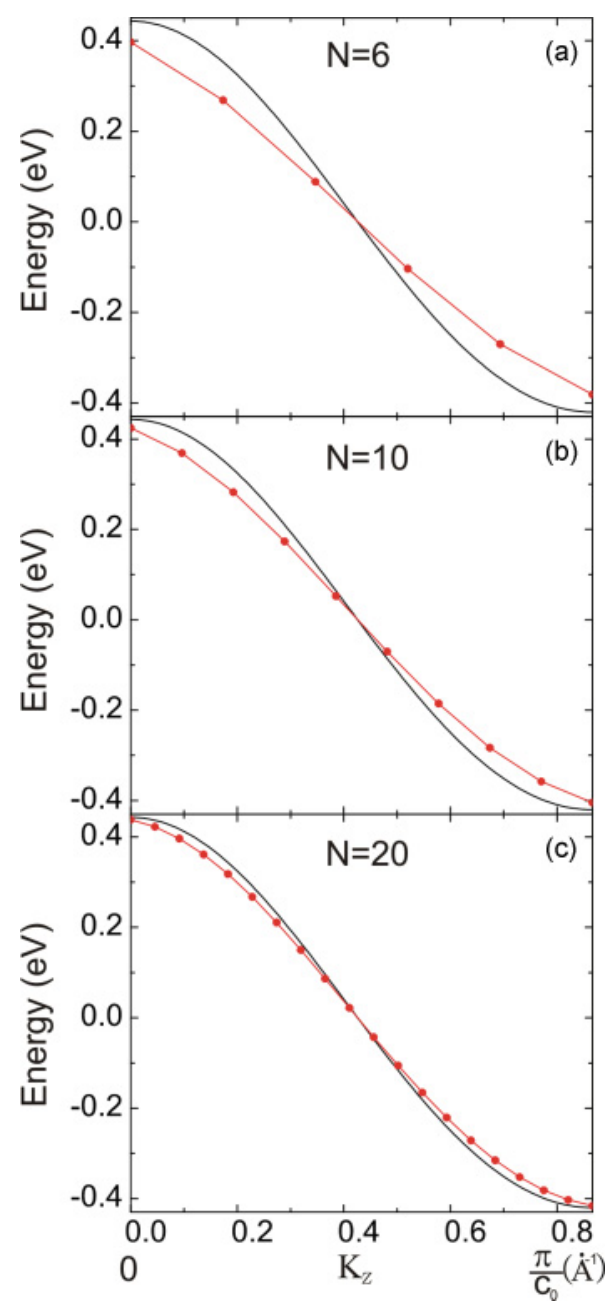

FIG. 5. (Color online) The sorted energy levels of (a) 6, (b) 10 , and (c) 20 AA-stacked graphene layers at the $K$ point, plotted equidistantly over the interval $\left[0, \pi / c_{0}\right]$.

overlap differs less than $10 \%$ with the band overlap of $4 \gamma_{1}$ in AA-stacked graphite. This property thus converges a bit faster than in AB-stacked graphite.

As the existence of multiple Dirac particles with different effective speeds of light is a particular property of AA-stacked graphite, it is also interesting to study the evolution of these velocities as function of the number of stacked layers. As shown above, the effective speeds of light, determined at the crossings of the bands, are good approximations for these velocities at the Fermi level. Therefore we compare in Fig. 7 the histogram distributions of the effective speeds at the $K$ point with the distribution Eq. (5) in AA-stacked graphite for 10, 20, and 30 layers in the interval $\left[c=\frac{\alpha}{\hbar}\left(\gamma_{0}+2 \gamma_{4}\right)=\right.$ $\left.8.20 \times 10^{5} \mathrm{~m} / \mathrm{s}, c=\frac{\alpha}{\hbar}\left(\gamma_{0}-2 \gamma_{4}\right)=8.47 \times 10^{5} \mathrm{~m} / \mathrm{s}\right]$, which are the two limiting effective speeds of light in AA-stacked graphite. From these histograms it is indeed clear that the distribution of these velocities evolves to the graphite limit. For a small number of layers, first particles with intermediate values for the effective speed of light are present. With increasing number of layers, more and more particles with effective speeds of light close to the two extremal values of bulk graphite appear. To quantify this evolution, we also plot

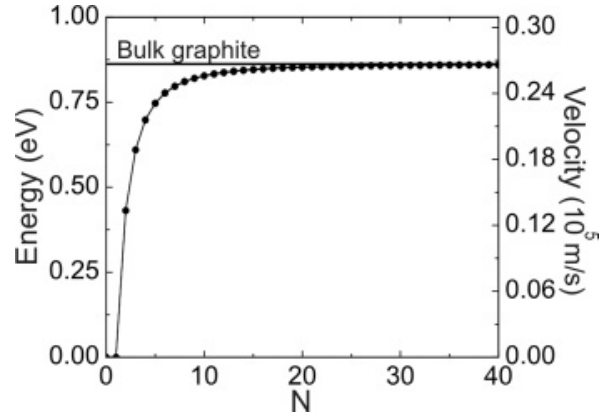

FIG. 6. Left scale: The energy difference between the last and first energy levels at the $K$ point which build up the graphite band for $N$ AA-stacked graphene layers. Right scale: The velocity difference between the maximal and minimal effective speed of light values at the $K$ point. The solid horizontal curve is the limiting value for bulk graphite: $4 \gamma_{1}$ for the energy difference and $2 \sqrt{3} a \gamma_{4} / \hbar$ for the velocity difference.

in Fig. 7 the obtained effective speeds of light equally spaced in the interval $\left[0, \pi / c_{0}\right]$, together with the velocities of Eq. (4). Again it is clear that the difference between the maximal and minimal values of the effective speed of light is a good measure for characterizing the deviation from the graphite limit. We plot this difference also in Fig. 6 and it is exactly the same as the energy difference studied above, but with a different scale.

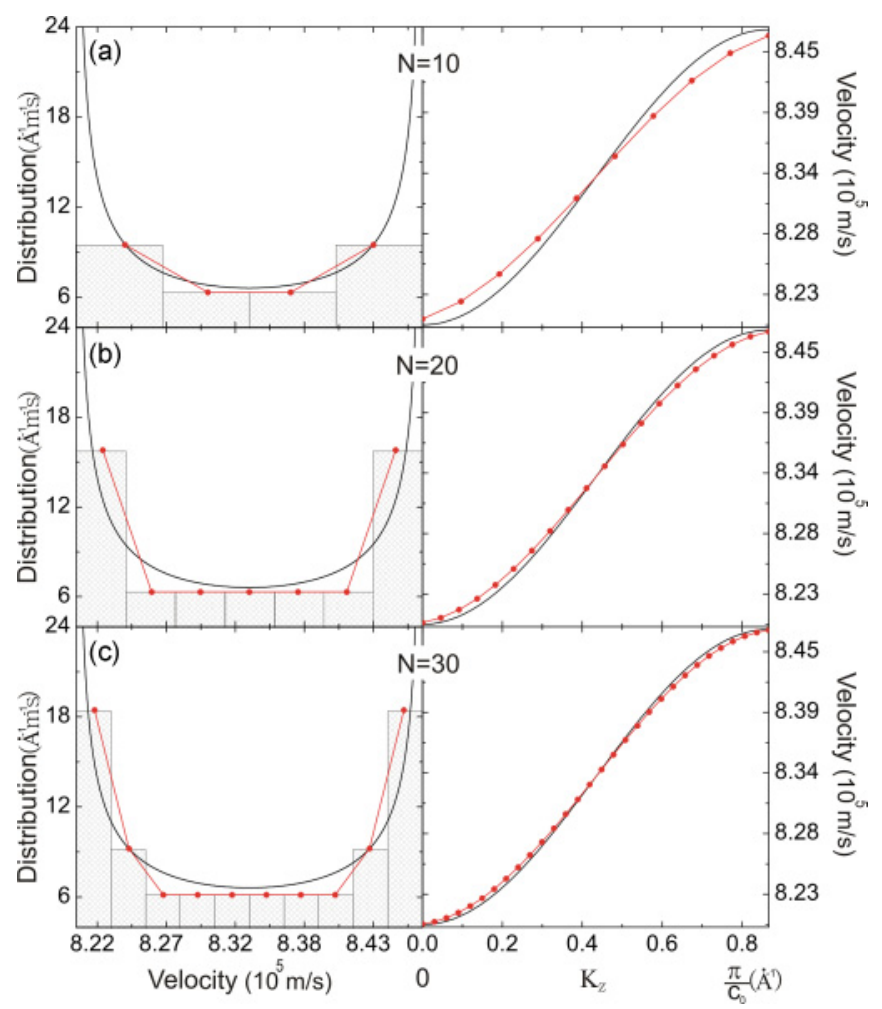

FIG. 7. (Color online) Left column: The distribution of the effective speeds of light at the $K$ point (red dots) compared to the distribution in AA-stacked graphite (full black curve). Right column: The sorted effective speeds of light at the $K$ point, plotted equidistantly over the interval $\left[0, \pi / c_{0}\right]$. The results are given for (a) 10 , (b) 20 , and (c) $30 \mathrm{AA}-$ stacked graphene layers. 

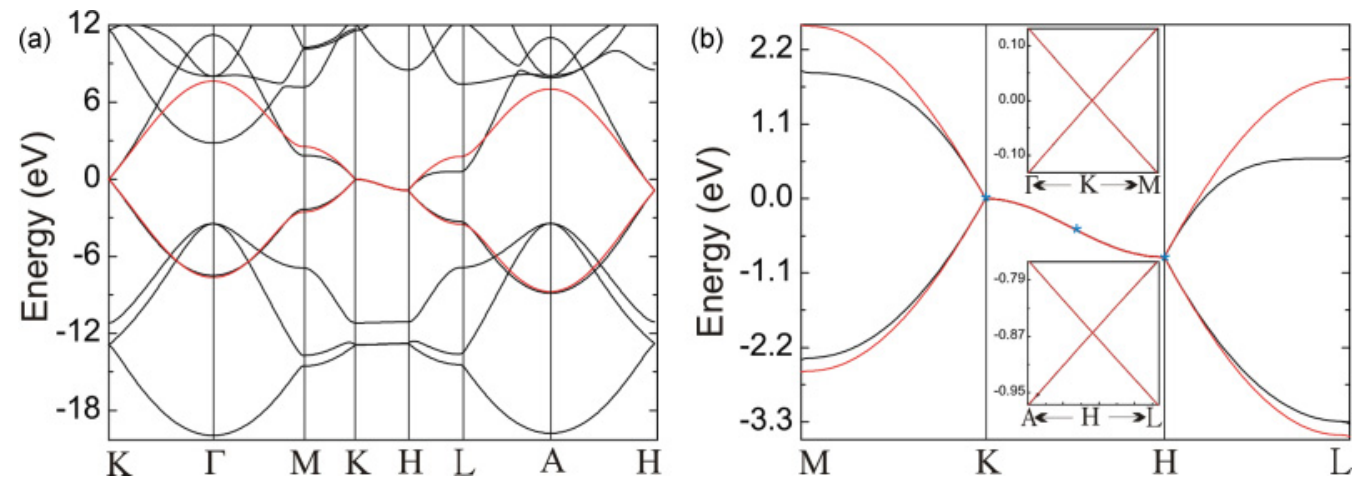

FIG. 8. (Color online) Ab initio (black curves) and tight-binding (blue curves) band structure of AA-stacked graphite (a) along $K \Gamma M K H L A H$, and (b) along $M K H L$. Insets show the energy dispersion around the $K$ and $H$ points.

\section{CONCLUSIONS}

We have investigated the electronic properties of the recently experimentally realized AA-stacked graphene multilayers and graphite using the tight-binding approach. The tightbinding parameters were obtained by first-principles density functional theory calculations. We find that in AA-stacked graphite there are no normal charge carriers present with a parabolic spectrum as in AB-stacked graphite, but all states at the Fermi energy along the $H K H$ line (in the direction of the $A \Gamma A$ line) have a linear spectrum with a different effective speed of light value, ranging from $c=\frac{\alpha}{\hbar}\left(\gamma_{0}+2 \gamma_{4}\right)=8.20 \times$ $10^{5} \mathrm{~m} / \mathrm{s}$ at the $K$ point to $c=\frac{\alpha}{\hbar}\left(\gamma_{0}-2 \gamma_{4}\right)=8.47 \times 10^{5} \mathrm{~m} / \mathrm{s}$ at the $H$ point. These velocities are not equally distributed but are peaked at these two extremal values at the $H$ and $K$ points. As the charge carrier pockets in AA-stacked graphite are located around these $K$ and $H$ points, we find that there exist in essence two types of massless Dirac particles in AA-stacked graphite. While the spectrum of two AB-stacked graphene layers is fundamentally different from the spectrum of a single graphene layer at the Fermi energy around the $K$ point, this is not the case for two AA-stacked graphene layers. The spectrum of two AA-stacked layers can be considered as a superposition of two single-layer spectra, and also only particles with a linear dispersion are present at the Fermi energy around the $K$ point. As the type of charge carriers in an AA-stacked bilayer differs fundamentally from the ones in an AB-stacked bilayer, it must be possible to discriminate between them using optical experiments. The AA-stacked graphene system is probably also the system most similar to pure graphene in which a gap may be created by applying an external electric field.

Increasing the number of AA-stacked graphene layers always changes the electronic spectrum in a similar way. AA-stacked graphite has a band overlap around $0.86 \mathrm{eV}$. It is shown that starting from 6 AA-stacked graphene layers, the band overlap differs less than $10 \%$ from this bulk value. This convergence to the bulk value is thus a bit faster than in the $\mathrm{AB}$ stacking case. It is also shown how the distribution of the effective speeds of light evolves to the bulk distribution. For a small number of layers, first particles with intermediate values of the effective speed of light are present, while more and more particles with effective speeds of light close to the two extremal values of bulk graphite appear if the number of layers increases. The difference between the maximal and minimal value of the present effective speed of light differs again less than $10 \%$ starting from 6 stacked AA levels.

\section{APPENDIX: TIGHT-BINDING PARAMETERS OBTAINED BY AB INITIO CALCULATIONS}

The presented tight-binding models include the parameters $\gamma_{0}, \gamma_{1}, \gamma_{4}$, and $\gamma_{5}$. These parameters are defined as in Refs. 14 and 15. To obtain these parameters $\gamma_{i}$ we compared the electronic structure obtained within the tight-binding approximation with $a b$ initio calculations. The band structures were calculated using the ABINIT density functional theory code, ${ }^{16}$ within the local density approximation (LDA). Although the LDA does not describe properly the van der Waals force, it has been proven to give a good prediction of the interlayer distance of AB-stacked graphite. We used Troullier-Martins norm-conserving pseudopotentials for the $2 s^{2} 2 p^{2}$ electrons of carbon, ${ }^{17}$ and a plane wave basis set with an energy cutoff of 40 hartrees was taken. Brillouin Zone integration was based on a $25 \times 25 \times 25$ and a $25 \times 25 \times 1$ Monkhorst-Pack grid $^{18}$ for AA-stacked graphite and multilayers of graphene, respectively. After structural optimization the cell parameter was found to be equal for all the structures: $a=2.45 \AA$, corresponding with a carbon-carbon distance equal to $1.41 \AA$, which is less than $0.5 \%$ from the experimental value.

The parameters $\gamma_{i}$ are obtained by solving analytically the tight-binding Hamiltonian for AA-stacked graphite and multilayers of graphene (1,2, and 3 layers) and then comparing the obtained energy and its derivative at the $K$ point with the density functional theory results.

\section{Graphite}

The Hamiltonian for AA-stacked graphite in the tightbinding approach near the $K$ point (without considering overlap integrals) and taking into account only $\pi$ bands is given by ${ }^{10}$

$$
H=\left[\begin{array}{cc}
e_{0}+\gamma_{1} \Gamma+\gamma_{5}\left(\Gamma^{2}-2\right) & f(\mathbf{k})\left(\gamma_{0}+\gamma_{4} \Gamma\right) \\
f^{*}(\mathbf{k})\left(\gamma_{0}+\gamma_{4} \Gamma\right) & e_{0}+\gamma_{1} \Gamma+\gamma_{5}\left(\Gamma^{2}-2\right)
\end{array}\right],
$$



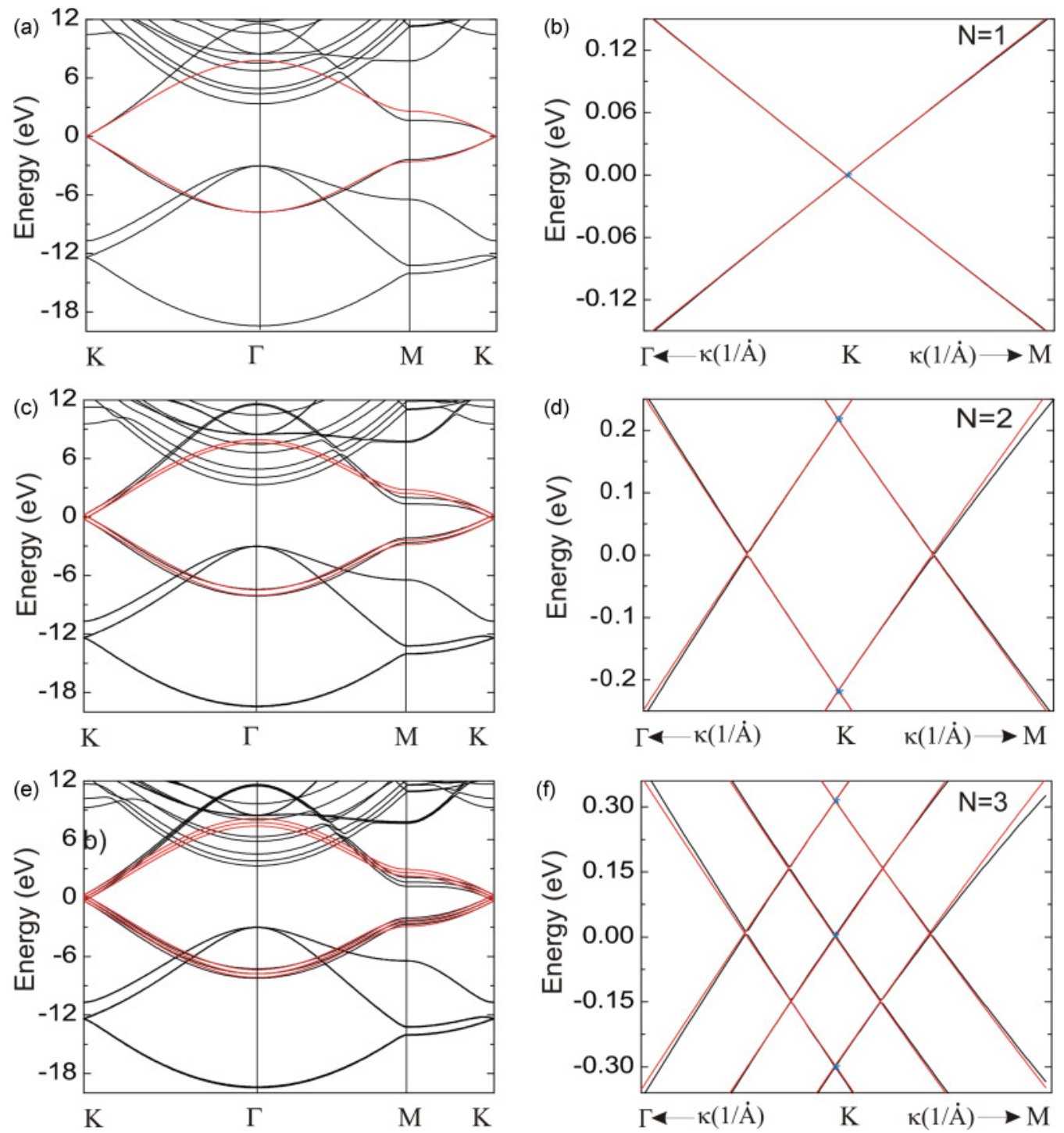

FIG. 9. (Color online) Ab initio (black curves) and tight-binding (blue curves) band structure along $K \Gamma M K$ of (a) graphene, (c) AA-stacked bilayer, and (e) AA-stacked trilayer. Corresponding energy dispersion around the $K$ point for (b) graphene, (d) AA-stacked bilayer, and (f) AA-stacked trilayer.

with eigenvalues

$$
E(\mathbf{k})=\left\{\begin{array}{l}
e_{0}+\gamma_{1} \Gamma+\gamma_{5}\left(\Gamma^{2}-2\right)+|f(\mathbf{k})|\left(\gamma_{0}+\gamma_{4} \Gamma\right), \\
e_{0}+\gamma_{1} \Gamma+\gamma_{5}\left(\Gamma^{2}-2\right)-|f(\mathbf{k})|\left(\gamma_{0}+\gamma_{4} \Gamma\right) .
\end{array}\right.
$$

At the $H K$ points the energy and its derivative are given by

$$
\begin{gathered}
E_{K}=e_{0}+2 \gamma_{1}+2 \gamma_{5}, \quad E_{K H / 2}=e_{0}-2 \gamma_{5}, \\
E_{H}=e_{0}-2 \gamma_{1}+2 \gamma_{5}, \quad V_{K}=\alpha\left(\gamma_{0}+2 \gamma_{4}\right), \\
V_{H}=\alpha\left(\gamma_{0}-2 \gamma_{4}\right) .
\end{gathered}
$$

Solving the equation system the tight-binding parameters in the $K$ point are

$$
\begin{gathered}
e_{0}=\left(E_{K}+E_{H}+2 E_{K H / 2}\right) / 4, \quad \gamma_{0}=\left(V_{K}+V_{H}\right) /(2 \alpha), \\
\gamma_{1}=\left(E_{K}-E_{H}\right) / 4, \quad \gamma_{4}=\left(V_{K}-E_{H}\right) /(4 \alpha), \\
y_{5}=\left(E_{K}+E_{H}-2 E_{K H / 2}\right) / 8 .
\end{gathered}
$$

After optimization of the unit cell of AA-stacked graphite, it was found that its lattice parameters are $a=b=2.45 \AA$ and $c=3.63 \AA$. Figure 8 shows the $a b$ initio (black curves) and tight-binding (blue curves) structures of AA-stacked

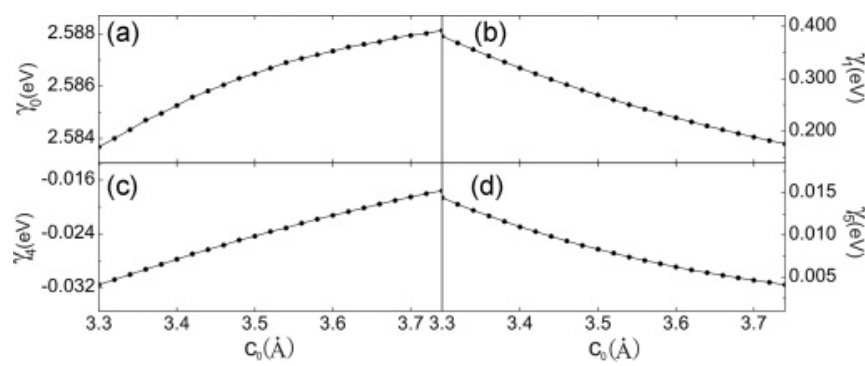

FIG. 10. Tight-binding parameter as a function of the interlayer separation for AA-stacked graphite: (a) $\gamma_{0}$, (b) $\gamma_{1}$, (c) $\gamma_{4}$, and (d) $\gamma_{5}$. 
graphite along $K \Gamma M K H L A H$ [Fig. 8(a)] and along $M K H L$ [Fig. 8(b)]. $A b$ initio and tight-binding calculations show very good agreement throughout the $K H$ line and even for the bonding $\pi$ band. The blue dots show the 3 points that were taken into account to calculate the tight-binding parameters.

\section{Graphene and AA-stacked bilayer and trilayer}

From the tight-binding Hamiltonians for graphene and the AA-stacked graphene bilayer and trilayer of Eq. (9), we obtain the eigenvalues (where an extra energy reference $e_{0}$ was introduced)

$$
\begin{aligned}
& E_{\text {graphene }}(\mathbf{k}) \\
& =\left\{\begin{array}{l}
e_{0}+\gamma_{0}|f(\mathbf{k})| \\
e_{0}-\gamma_{0}|f(\mathbf{k})|
\end{array}\left|\begin{array}{l}
E_{\text {bilayer }}(\mathbf{k}) \\
e_{0}+\gamma_{0}|f(\mathbf{k})|-\gamma_{1}+\gamma_{4}|f(\mathbf{k})| \\
e_{0}-\gamma_{0}|f(\mathbf{k})|-\gamma_{1}-\gamma_{4}|f(\mathbf{k})|
\end{array}\right| \begin{array}{l}
e_{0}+\gamma_{0}|f(\mathbf{k})|+\gamma_{1}+\gamma_{4}|f(\mathbf{k})| \\
e_{0}|f(\mathbf{k})|+\gamma_{1}-\gamma_{4}|f(\mathbf{k})| \\
e_{0}-\gamma_{5}-\gamma_{0}|f(\mathbf{k})|
\end{array} \mid \begin{array}{l}
e_{0}+\frac{\gamma_{5}}{2}+\gamma_{0}|f(\mathbf{k})|+\frac{\sqrt{\gamma_{5}^{2}+8\left(\gamma_{1}-\gamma_{4}|f(\mathbf{k})|\right)^{2}}}{2} \\
e_{0}+\frac{\gamma_{5}}{2}-\gamma_{0}|f(\mathbf{k})|+\frac{\sqrt{\gamma_{5}^{2}+8\left(\gamma_{1}-\gamma_{4}|f(\mathbf{k})|\right)^{2}}}{2} \\
e_{0}+\frac{\gamma_{5}}{2}+\gamma_{0}|f(\mathbf{k})|-\frac{\sqrt{\gamma_{5}^{2}+8\left(\gamma_{1}-\gamma_{4}|f(\mathbf{k})|\right)^{2}}}{2} \\
e_{0}+\frac{\gamma_{5}}{2}-\gamma_{0}|f(\mathbf{k})|-\frac{\sqrt{\gamma_{5}^{2}+8\left(\gamma_{1}-\gamma_{4}|f(\mathbf{k})|\right)^{2}}}{2}
\end{array}\right.
\end{aligned}
$$

At the $K$ point the energies and their derivatives are given by

$$
\begin{array}{l|l|l} 
& & E_{u}=e_{0}+\left(\gamma_{5}+\sqrt{\gamma_{5}^{2}+8 \gamma_{1}^{2}}\right) / 2 \\
E_{K}=e_{0} & E_{u}=e_{0}+\gamma_{1} & E_{0}-\gamma_{5} \\
V_{K}=\alpha \gamma_{0}=e_{0}-\gamma_{1} & E_{d}=e_{0}+\left(\gamma_{5}-\sqrt{\gamma_{5}^{2}+8 \gamma_{1}^{2}}\right) / 2 \\
V_{u}=\alpha\left(\gamma_{0}+\gamma_{4}\right) & V_{u}=\alpha\left(\gamma_{0}+4 \gamma_{1} \gamma_{4} / \sqrt{\gamma_{5}^{2}+8 \gamma_{1}^{2}}\right) \\
V_{d}=\alpha\left(\gamma_{0}-\gamma_{4}\right) & V_{m}=\alpha \gamma_{0} \\
& V_{d}=\alpha\left(\gamma_{0}-4 \gamma_{1} \gamma_{4} / \sqrt{\gamma_{5}^{2}+8 \gamma_{1}^{2}}\right)
\end{array}
$$

Therefore, the tight binding parameters are given by

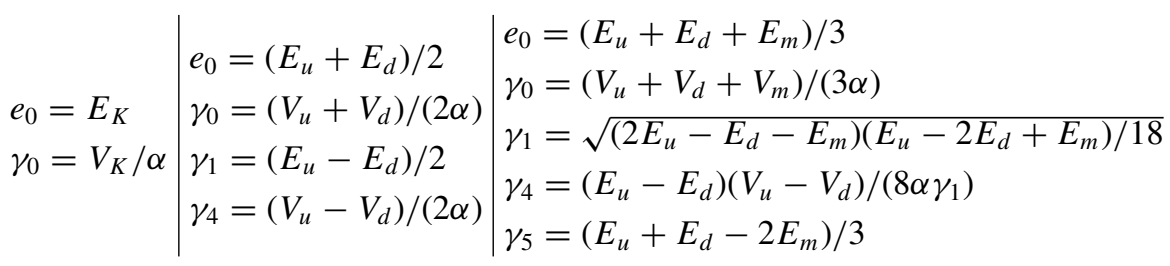

Figure 9 shows the $a b$ initio (black curves) and tight-binding (red curves) band structure along $K \Gamma M K$ for graphene (a), the AA-stacked bilayer (c), and the AA-stacked trilayer (e). The energy dispersion around the $K$ point shows very good agreement between the $a b$ initio and tight-binding calculations [see Figs. 9(b), 9(d), and 9(f), respectively]. The blue dots show the points that were taken into account to get the tight-binding parameters.

\section{Dependence of the tight-binding parameters on the interlayer spacing}

To investigate the importance of a correct interlayer distance we investigated the dependence of the tight-binding parameters of AA-stacked graphite with respect to this interlayer separation by first-principles density functional theory calculations. The range covered was from $3.30 \AA$ to $3.74 \AA$. The dependence is shown in Fig. 10. All parameters show a small and almost linear dependence with respect to the interlayer spacing. *bart.partoens@ua.ac.be

${ }^{1}$ J. D. Bernal, Proc. R. Soc. London A 106, 749 (1924).

${ }^{2}$ R. R. Hearing, Can. J. Phys. 36, 352 (1958).

${ }^{3}$ L. Samuelson and I. P. Batra, J. Phys. C 13, 5105 (1980).
${ }^{4}$ J.-K. Lee, S.-C. Lee, J.-P. Ahn, S.-C. Kim, J. I. B. Wilson, and Ph. John, J. Chem. Phys. 129, 234709 (2008).

${ }^{5}$ Z. Liu, K. Suenaga, P. J. F. Harris, and S. Iijima, Phys. Rev. Lett. 102, 015501 (2009). 
${ }^{6}$ D. D. L. Chung, J. Mater. Sci. 37, 1475 (2002).

${ }^{7}$ K. S. Novoselov, A. K. Geim, S. V. Morozov, D. Jiang, Y. Zhang, S. V. Dubonos, I. V. Grigorieva, and A. A. Firsov, Science 306, 666 (2004).

${ }^{8}$ V. P. Gusynin and S. G. Sharapov, Phys. Rev. Lett. 95, 146801 (2005).

${ }^{9}$ J.-C. Charlier, J.-P. Michenaud, and X. Gonze, Phys. Rev. B 46, 4531 (1992).

${ }^{10}$ J.-C. Charlier, J.-P. Michenaud, X. Gonze, and J.-P. Vigneron, Phys. Rev. B 44, 13237 (1991).
${ }^{11}$ C. L. Lu, C. P. Chang, and M. F. Lin, Eur. Phys. J. B 60, 161 (2007).

${ }^{12}$ C. L. Lu, C. P. Chang, Y. C. Huang, J. H. Ho, C. C. Hwang, and M. F. Lin, J. Phys. Soc. Jpn. 76, 024701 (2007).

${ }^{13}$ Y. Xu, X. Li, and J. Dong, Nanotechnology 21, 065711 (2010).

${ }^{14}$ B. Partoens and F. M. Peeters, Phys. Rev. B 74, 075404 (2006).

${ }^{15}$ J.-C. Charlier, J.-P. Michenaud, and Ph. Lambin, Phys. Rev. B 46, 4540 (1992).

${ }^{16}$ X. Gonze et al., Comput. Mater. Sci. 25, 478 (2002).

${ }^{17}$ N. Troullier and J. L. Martins, Phys. Rev. B 43, 8861 (1991).

${ }^{18}$ H. J. Monkhorst and J. D. Pack, Phys. Rev. B 13, 5188 (1976). 